\title{
Discussion to the papers by D. Hallberg et al.; and M. J. T. Peaston
}

Carlo. It is indeed a pleasure to review a paper as objective as Dr Peaston's. This work illustrates clearly the therapeutical and practical aspects of nutritional problems during intensive patient care. The validity of his experimental approach is demonstrated by the clear and useful conclusions he was able to draw. The title of the paper already stressed his comprehensive approach to nutrition. Indeed, the title 'Maintenance of Metabolism during Intensive Patient Care' and not simply 'Nutritional Problems during Intensive Patient Care' indicates immediately one particular aspect of Dr Peaston's approach that deserves special emphasis: the dependence on nutrition of the maintenance of metabolism and its direct repercussion on the internal environment as reflected in particular by the acid-base balance.

It is important to realize that the internal environment should be regarded as reflecting the functional conditions of intracellular metabolism. When imbalance is observed in the internal environment, therapeutic efforts should be made to bring intracellular metabolism back to normal. One of the main logical and effective measures, therefore, is to make sure that cells receive adequate substrates.

Another important point of Dr Peaston's approach is illustrated by his definition of the maintenance of metabolism during severe illness as being the provision of the observed or predicted need for calories, water, electrolytes, nitrogen and other essential anions in order to maintain as much as possible, or to approach as close as possible, the return to homeostasis.

Indeed, with the presently available nutritional means, particularly the nutritional solutions for intravenous feeding, imbalance due to the absence or the deficiency of essential nutrients should never be allowed to develop. Dr Peaston has stressed quite judiciously the provision of predicted needs.

The present study demonstrates clearly the benefits that can be derived from naso-gastric and parenteral feeding, and also the effectiveness of parenteral feeding when other routes are not available.

It cannot be over-emphasized that, during acute illness or following trauma, adequate nutrition is exceptional. Therefore, it is necessary to administer either by nasogastric tube or parenterally adequate calories and nitrogen in order to preserve a satisfactory nitrogen balance.

The catabolic response to trauma should be reversed as soon as possible; it is unphysiological and largely due to calorie restriction associated with the inadequacy of traditional intravenous infusions.

For the supply of nutritional elements, it should be mentioned that, although fats supply a maximum amount of calories per gram, carbohydrates are the only immediately utilizable energy source and a proper proportion between the calories provided by fat and by carbohydrates should be given for the best utilization. Glycogen reserves are normally around 300-400 g. Following trauma, these stores can be exhausted within 6-8 $\mathrm{hr}$. The organism then has to call on gluconeogenesis at the expense of proteins to maintain adequate carbohydrate metabolism, and this leads to severe nitrogen loss. There is nothing miraculous about the beneficial effects of fats, carbohydrates and amino acids, whose benefits can be explained on the basis that they are mainly substrates without which the cell cannot function adequately.

TAYLOR. I wish to disagree with Dr Peaston's conclusion that the obligatory catabolic breakdown of protein in response to trauma may be reversed by feeding what are essentially diets of normal nitrogen and caloric content. I do not think his results establish this point at all.

Let us begin with the controls. It is well known that a normal, healthy adult person who rests in bed for a week or so, goes into negative nitrogen balance. Even if he is given a high protein diet his muscle mass still diminishes, as a result of the prolonged inactivity. He may gain weight, as fat, but not muscle mass. Dr Peaston refers to 'healthy active controls', who were presumably therefore not in bed. His control data thus show no more than that 1950 calories were inadequate to maintain normal weight and that 2580 calories were an improvement on this. A previously healthy, adult patient will also, of course, lose muscle mass and nitrogen, by simply being in bed and inactive. It is difficult to believe that this loss can be reversed in patients when it cannot be in normal subjects.

With regard to the patients-only one of those who were tube-fed had experienced trauma other than tracheostomy, although about half had pulmonary sepsis. Of those fed intravenously only three appear to have experienced trauma per se, yet thirteen (out of eighteen) had malnutrition. Now, as Dr Peaston points out, malnourished patients do not exhibit the obligatory catabolic breakdown of protein after trauma-that is to say, they increase their depleted muscle mass readily when given protein, despite coincident trauma. It is, of course, quite correct to feed such patients adequately, as Dr Peaston has done, but it is wrong to conclude that the obligatory protein loss is being thereby overcome, because it would not have occurred anyway and because in a traumatized, previously-healthy adult an obligatory loss would still have been found. I feel that if Dr Peaston were to classify his patients into traumatic, infective and malnourished groups he would find the same significant differences between them that others have in the past. Certainly it is necessary to divide them up in this way if he is to convince us to the contrary.

Whenever I try to feed patients with high protein or amino acid intakes in the first few days after trauma, I invariably find that I push an already raised blood urea even higher. This seems to indicate that the exogenous protein is catabolized. It also seems wrong to raise the blood urea in this way before one can be sure that a further surgical or traumatic episode may not be needed, with its attendant risk of acute renal failure. It would be interesting to know what blood urea levels were attained in the 'traumatic' patients of Dr Peaston. It is amazing how few of the papers dealing with nitrogen balances after trauma give data for the blood urea.

The time after injury or sepsis is, of course, all important in the assessment of metabolic balances. After about the 5th day, the repair phase begins and nitrogen balances become strongly positive. Dr Peaston says that 'no patient presented had balance data compiled for less than 4 days' and the mean was 8.3 days. It seems clear, therefore, that a part of the balance study in each of the 'traumatic' and 'infective' patients would have been 
carried out during the anabolic phase. Again, therefore, the method of expressing the results would mask the changes which have been so carefully established by others since 1932. Again I would ask Dr Peaston to sub-divide his results, say into 4-day periods after trauma, and see if his experience is not really that of his predecessors.

Lastly, we must understand fully what it is, on biochemical grounds, that Dr Peaston is asking us to accept: that exogenous protein or amino acids will be utilized anabolically more effectively than the body's own protein and amino-acids which would otherwise be lost, and conversely, that the latter will be turned more readily to urea in the liver than will the former.

Peaston. Dr Taylor's observations are most stimulating, and I should like to deal with them in detail. I quite agree that the comparison between the two tubefeeds in healthy active controls proves no more than that the second diet was an improvement on the first. But as this was all it was intended to prove anyway, it has still fulfilled its purpose. What is more to the point is that the modified diet was shown to preserve metabolic balance in serious illnesses.

Of the patients treated by tube feeding Dr Taylor points out that only one patient had suffered serious trauma. This is quite true, but my studies were not designed to apply solely to patients who had suffered surgery or trauma, but to apply to all patients admitted to an Intensive Care Unit because their illness warranted it. As Dr Taylor indicates, trauma constitutes a small proportion of the types of case admitted to the Unit in which these studies were performed. I don't believe, however, that anyone who analyses the twenty consecutive unselected patients reported would deny that they represent a group with very serious illnesses. The fact that metabolic balance was preserved as effectively as the balance results unequivocally show is in my view sufficient vindication of the method employed.

With respect, Mr Chairman, I feel Dr Taylor has misunderstood the purpose of the balance experiments conducted on the intravenous materials. The experiments were designed for the sole purpose of establishing a diet of fixed composition which is suitable for application in an Intensive Care Unit to those patients who cannot be fed by any other route. Clearly it was ethically impossible to use healthy controls for these experiments. The three diets tested were therefore given to three comparable groups of patients in whom a period of intravenous nutrition was considered clinically necessary. Each group contains one patient with radical cancer surgery, two patients with carcinoma, and four with malnutrition. The diet chosen was that which produced the most satisfactory balance data.

Dr Taylor implies that my patients should be classified into pathological groups. I submit that this is quite irrelevant, since the patients arriving at the doors of an Intensive Care Unit don't, unfortunately, group themselves into convenient platoons with similar pathologies. The aim has been to devise feeding methods satisfactory for all. I readily accept that if the patients were grouped into traumatic, infective and malnourished groups significant balance differences would be apparent. But, as Dr Taylor says, these differences are already well known, and it has not been my purpose to teach what is already known to those who already know.

The claim that some patients would not be preserved in metabolic balance in severe trauma, sepsis, or both, $\vec{\equiv}$ is likewise one I readily concede. But this is no argument for supervised starvation. The point that these patients would exhibit no catabolic response is similarly irrelevant - the point is surely that, if not fed, they would be obliged to live on their own lean muscle mass, as does any subject who is systematically starved. The fact that they were fed prevented this.

In referring again to the tube-fed patients Dr Taylor implies that with some patients the balance data covered the 'catabolic' 5-day period, whereas in others the data include the subsequent days in which the 'anabolic' phase would give a positive nitrogen balance anyway, so that the data are unfairly weighted in favour of positive balance. In fact, of course, precisely the opposite is the case! Of the twenty patients studied for 166 balance days, no less than ninety-seven of those days were in the first 5-day period, or catabolic phase, of their illness, and only sixty-nine in the subsequent anabolic period. So that if anything the data are weighted towards the catabolic period in a ratio of $3: 2$. Once again, however, this is not really a relevant issue, since the methods are intended to supply optimum nutritional support throughout the whole of an individual patient's illness, and as patients do not have illnesses in convenient 5-day parcels, dividing their metabolic balance data into such arbitrary periods is quite unrealistic.

I was most interested to hear that Dr Taylor finds that giving patients a normal dietary nitrogen intake as intravenous amino-acids following trauma causes a further rise in an already raised blood urea. But why do his patients already have a raised blood urea? If they are not in renal failure they are presumably suffering from starvation and dehydration. I agree that the starved and dehydrated should have their fluid balance corrected before amino-acids are infused, and that those in renal failure should not receive infusions of amino acids unless dialysis is programmed. All the patients I have fed with intravenous amino acids have had frequent blood urea estimations, and have shown no elevation of blood urea. The small variations have been taken into account in compiling the 'corrected' nitrogen balance data. Patients with a raised blood urea due to extrarenal fluid and electrolyte depletion can, however, be safely infused with amino acids provided adequate fluid and electrolytes are also given. I can recall two patients with blood ureas of 200 and $118 \mathrm{mg} / 100 \mathrm{ml}$ due to dehydration, and who received intravenous amino acids in generous amounts along with adequate fluid replacement and whose blood ureas fell to 30 and $27 \mathrm{mg} / 100 \mathrm{ml}$ in 7 and 5 days respectively.

Finally, Mr Chairman, I would like to take issue with Dr Taylor on a purely philosophical level. My major complaint is that he appears rigidly convinced that the metabolic response to illness always has been, and always will be, both inevitable and irreversible. If this Symposium has made any one single point, it is that Intensive Care necessarily includes a challenge to the rigid beliefs and fatalistic defeatism of conventional medicine. Dr Nixon has challenged the inevitability of death from cardiac shock. Dr Riding has challenged the inevitable outcome in the moribund asthmatic. I am challenging the passive 
acceptance that the desperately ill should be allowed to live on their own muscle protein by a process of endogenous cannibalism. I hope I have made my point.

LEE. I should like to emphasize one or two points arising from Dr Hallberg's contribution. Everyone has had the experience of seeing a difficult surgical or medical case successfully navigated through an early crisis only later to become apathetic, anorexic and progressively more emaciated, often eventually to die, in many intances from what appears to be an unknown cause. It is a sad thought that still in 1966 many patients die in British Hospitals from nothing less than starvation. We have all heard our colleagues, and some of us might be likewise guilty, say when questioned about the nutrition of their patients, that he is receiving either glucose saline or indeed normal saline. May I remind you that a litre of $4.2 \%$ dextrose contains but 180 calories and that 1 litre of $10 \%$ fructose contains 400 calories and, dare I say it, that normal saline contains none. An effective means of providing complete parenteral nutrition is now available but it seems still inadequately used. There are now available, as we have just heard, a number of fatemulsion preparations and amino-acid-containing preparations. When using parenteral nutrition it must not be thought of as merely providing calories but rather as a means of giving a balanced intravenous diet and attempting to maintain metabolic equilibrium. For this purpose it is necessary to know the patient's weight and to know what the mean daily requirement of nitrogen, carbohydrate and fat is for a given patient and then by juggling with the appropriate volumes of the large variety of solutions now available, a complete diet can be given intravenously. A few of these solutions contain electrolytes and electrolytes have to be added to others. I have personally over the past 3 years used over a thousand infusions of amino-acid-containing solutions and fat emulsions with no serious untoward side effects either acutely or chronically. Further, in those patients who have died from their primary disease whilst receiving such treatment, no pathological findings attributable to the intravenous nutrition therapy have been found. I have kept patients going for as long as 6 weeks by this means and I know that Mr Lawson has coped with such patients for as long as 12 weeks, without any untoward side effects. Not only do these patients gain weight, seem less prone to infection, have shorter hospital stay times, but also, which is much more difficult to measure, they appear and feel very much better. One small point to remember is that most of these solutions do not contain vitamins and thus these need to be added to any such parenteral nutrition regime. I should add that my own experience has been entirely with soya bean fat emulsions and casein hydrolysates. The incidence of thrombophlebitis following such therapy has been cited as a possible difficulty in this treatment. I have rarely come across this complication but it can usually be avoided by administering the fat emulsion and amino-acid solution simultaneously through the giving needle. I have devoted considerable time to the application of such solutions and emulsions in the management of acute renal failure patients, who could not take oral nutrition. Several reports have appeared suggesting that the administration of fat emulsions either immediately prior to or during haemodialysis interferes with the clearance characteristics of the artificial kidney membrane. I have carefully studied this problem and can state quite categorically that emulsions do not alter the clearance characteristics of the membrane whether in a Kolff twin-coil disposable kidney or Kiil artificial kidney. The addition of these solutions to the nephrologists' therapeutic armamentarium has made the management of hypercatabolic acute renal failure very much easier and has greatly reduced morbidity.

CARLO. Dr Hallberg's presentation does not bring out some important details of the experimental procedure followed. The dogs given the alternative fat preparations were all underweight when compared with those given Intralipid. It is unjustified to draw the conclusion that the cottonseed oil emulsions studied are toxic merely because they contain cottonseed oil, when in fact it has been demonstrated that the synthetic emulsifiers present in the preparations are responsible for this toxicity. An emulsion prepared with soya bean oil and this type of emulsifier would be just as toxic. Consequently, the comparative studies presented cannot be regarded as decisive. The only fat emulsion which has been made available in the United States, but now subsequently, withdrawn by the F.D.A., contained a synthetic emulsifier. It does not correspond with the formula of the cottonseed oil emulsion Lipiphysan, which is in use in England and on the Continent.

Brooks. We have found that when pure synthetic amino acids are administered intravenously together with sorbitol the major part of the infusion is retained and in some patients the plasma-protein concentration rises. The preparation is well tolerated and no abnormal sensitivity reaction have so far been noted. Except in the more concentrated forms, venous thrombosis has not been a problem.

Various types of patients have received the infusion. These have included patients who have suffered head injury and craniotomy, gastric surgery, or open heart surgery with infection. A positive nitrogen balance has been obtained in some and the valuable part played by the amino-acid preparations has been demonstrated. It would appear to me that when infused in balanced proportions, synthetic amino-acid preparations are valuable in preventing major protein deficits occurring in postoperative patients in whom complications have developed and the catabolic phase is prolonged.

The fact that, in the higher animals, the naturally occurring amino acids are in the $l$-form and synthetic amino acids are in the racemic form does not appear to markedly affect utilization in the post-operative patient. There is increasing evidence that a number of aminoacids in the $d$-form can be incorporated into tissue protein, because the corresponding $l$-form can be recovered by using isotope tracers. It is possible to predict the existence of amino-acid isomerases.

It has also been suggested that sorbitol is not readily metabolized. This has certainly not been our experience. The maximum excretion of sorbitol we have obtained was $16 \%$ of the total quantity infused and in the majority of patients only 4-5\%was excreted. From our own balance studies it appears that the combination of sorbitol with amino-acids is a convenient method of intravenous nutrition which can be used in the presence of postoperative jaundice, infection or cardiac failure. 
TAYLOR. Although we have not had the extensive experience of the previous speakers, I can confirm that in the Liverpool Royal Infirmary we have not found 'Intralipid' to have toxic properties when infused in amounts of $500 \mathrm{ml}$ of the $20 \%$ emulsion daily, and when given for periods of up to 4 weeks. I should like to ask if it is not the case that the use of cottonseed oil is forbidden in the U.S.A.?

Hallberg. In answer to Dr Taylor's question concerning why the soyabean oil emulsion is less toxic than the cottonseed oil emulsion: The two fat emulsions differ in three respects. First: they are made from vegetable oils. Secondly, different emulsifiers are used and, lastly, there are different manufacturers.

The two oils have never been studied under exactly similar conditions, viz. with the same emulsifiers, the same manufacturer and the same investigator. For these reasons there is at the moment no true answer to your question why the cottonseed oil emulsion is more toxic than the soyabean oil emulsion. The difference may depend on any of these factors.
HoBss. (Sépépharm, Ltd): As a non-medical contributor I should like to make a comment, since I believe I can answer Dr Taylor's question concerning the use of the cottonseed oil emulsions in the United States. The first intravenous fat emulsion to achieve any degree of success was Lipomul, which was withdrawn by the Food and Drugs Administration of the U.S.A. some time ago. This was followed by a preparation called Infonutrol which was withdrawn by the manufacturers in April 1966. Both these products consisted of cottonseed oil emulsified with pluronics, and, because of this, or because the cottonseed oil contained gossypol, they were both found to be toxic.

The two i.v. fat emulsions now available in Great Britain are Intralipid (soyabean oil) and Lipiphysan (cottonseed oil). Lipiphysan contains neither gossypol nor pluronics.

TAYLOR. Are there any fat emulsions approved by the F.D.A.?

HoBbs. To the best of my knowledge, there are no fat emulsions commercially available in the U.S.A. 